Pacific

Journal of

Mathematics

CONFLUENCE $q$-DIFFÉRENCE VERS DIFFÉRENCE POUR UN SYSTÈME FUCHSIEN

Anne Duval

Volume $217 \quad$ No. 2

December 2004 


\title{
CONFLUENCE $q$-DIFFÉRENCE VERS DIFFÉRENCE POUR UN SYSTÈME FUCHSIEN
}

\author{
AnNe Duval
}

\begin{abstract}
In a previous article we showed how one can see the classical result on existence and uniqueness of a fundamental set of factorial series solutions of a regular difference system as the limit, when $q$ tends to 1 , of analogous results for a $\boldsymbol{q}$-difference system obtained by some kind of deformation. We extend here this property to singular-regular (or Fuchsian) systems.
\end{abstract}

\section{Introduction}

Il est bien connu qu'une équation aux $q$-différences (resp. aux différences de pas $\varepsilon$ ) dégénère en une équation différentielle lorsque $q \rightarrow 1$ (resp. $\varepsilon \rightarrow 0$ ) et que ce phénomène se traduit au niveau des solutions. Cette confluence, étudiée de longue date, a été reprise récemment dans le cas des $q$-différences par J. Sauloy qui en propose une théorie précise et complète dans [7]. L'étude parallèle du cas différence n'est pas faite et semble plus difficile. C'est un autre type, probablement plus abordable, de confluence que nous envisageons ici, la dégénérescence d'une équation aux $q$-différences en une équation aux différences. Il s'agit dans les deux cas d'une équation fonctionnelle construite à partir d'une homographie, à deux points fixes dans le premier cas, un seul dans le second cas. Dans [1] nous abordions, dans l'esprit de [7], le cas régulier. Nous y montrions comment on peut voir le classique théorème d'existence et d'unicité d'un système fondamental de solutions développables en séries de factorielles convergentes et à valeur prescrite en $+\infty$ comme limite, lorsque le paramètre réel $q$ tend vers $1^{+}$, de résultats analogues pour un système aux $q$-différences qui en est une déformation convenable. Nous étudions ici le cas d'un système aux différences singulierrégulier (ou fuchsien) au sens de $[3]$ ou [6]. Nous utilisons en particulier une famille de "caractères" inspirée de celle qu'utilise J. Sauloy ([7]) dans le cas $q$-différence.

Indiquons le plan de l'article. Dans un premier paragraphe, nous rappelons ce qu'on entend par système aux différences fuchsien puis indiquons comment le déformer en un système aux $q$-différences également fuchsien. Le deuxième paragraphe est consacré au cas d'un système à coefficients constants. On y définit en particuler une famille de caractères méromorphes 
dans C. Le dernier paragraphe montre enfin comment modifier et utiliser les résultats de [1] pour ramener le cas fuchsien au cas constant.

\section{Le cadre de l'étude}

A l'homothétie $\sigma_{q}(x)=q x$ est associé l'opérateur aux $q$-différences $\sigma_{q}(f)(x)$ $=f(q x)$. Si on remplace $\sigma_{q}$ par l'homographie $\sigma_{q, 1}(x)=q x+1$ puis que l'on fait tendre $q$ vers 1 , on obtient la translation de pas $1, \tau f(x)=f(x+1)$.

L'homographie $\sigma_{q, 1}$ a, comme $\sigma_{q}$, deux points fixes et une équation fonctionnelle faisant intervenir $\sigma_{q, 1}$ peut être transformée en une équation aux $q$-différences par un changement homographique de la variable. L'un des deux points fixes de $\sigma_{q, 1}$ est situé en $1 /(1-q)$ et conflue, lorsque $q \rightarrow 1$, vers l'autre, situé en $\infty$.

On peut alors étudier les solutions d'un système aux différences linéaire

$$
X(x+1)=A(x) X(x)
$$

où $A(x)$ est une matrice carrée de dimension $\mu$ et $X$ un vecteur de dimension $\mu$, à partir de celles d'une déformation

$$
X(q x+1)=A_{q}(x) X(x) .
$$

La forme de $A_{q}(x)$, vérifiant $\lim _{q \rightarrow 1} A_{q}(x)=A(x)$, est précisée au paragraphe 1.2, après un paragraphe de rappels concernant les systèmes aux différences singuliers réguliers (ou fuchsiens) en $+\infty$.

1.1. Système aux différences fuchsien en $+\infty$. La terminologie choisie est inspirée de celle du cas différentiel. Le parallélisme entre les deux théories est frappant si l'on utilise l'opérateur $(x-1){ }_{-1}^{\Delta}$ où

$$
\Delta_{-1}^{\Delta} f(x)=f(x)-f(x-1)
$$

et les séries de factorielles dont nous rappelons la définition.

On choisit une norme sur $\mathbf{C}^{\mu}$ et une norme compatible sur $g l(\mu, \mathbf{C})$, c'està-dire que l'on impose, pour $A \in g l(\mu, \mathbf{C})$ et $U \in \mathbf{C}^{\mu}$, la condition $\|A U\| \leq$ $\|A\|\|U\|$.

Définition 1.1. Pour tout entier $s \geq 0$, on pose

$$
x^{-[s]}= \begin{cases}\frac{1}{x(x+1) \cdots(x+s-1)} & \text { si } s \geq 1 \\ 1 & \text { si } s=0 .\end{cases}
$$

Soit $A(x)=\sum_{s \geq 0} A_{s} x^{-[s]}$ où $A_{s} \in g l(\mu, \mathbf{C})$ (resp. $A_{s} \in \mathbf{C}^{\mu}$ ) une série de factorielles (formelle). Si $C>0$ et $\lambda>0$ sont fixés, on dit que la matrice (resp. le vecteur) $A(x)$ vérifie la condition $(C, \lambda)$ lorsque pour tout $s \geq 1$, on a $\left\|A_{s}\right\| \leq C \frac{\Gamma(\lambda+s-1)}{\Gamma(\lambda)}$. 
La condition $(C, \lambda)$ assure la convergence (absolue) de la série $A(x)$ dans le demi-plan $\Re x>\lambda$. Sa somme est alors une fonction holomorphe dans le demi-plan de convergence. Toute fonction holomorphe à l'infini admet un développement en série de factorielles convergente dans un demi-plan $\Re x \gg 0$, mais la réciproque est fausse.

Définition 1.2. Le système $(*)$ est dit "de première espèce" s'il s'écrit

$$
(x-1){ }_{-1}^{\Delta} X(x)=A(x) X(x) .
$$

où $A(x)$ vérifie une condition $(C, \lambda)$.

Le résultat suivant est classique ([6] ou [3]).

Proposition 1.3. Un système de première espèce admet un système fondamental de solutions de la forme $\mathcal{X}(x)=\mathcal{F}(x) x^{R}$ où $R$ est une matrice constante, $\mathcal{F}(x)=\sum_{s \geq 0} F_{s} x^{-[s]}$ vérifie une condition $(\widetilde{C}, \widetilde{\lambda})$ et $F_{0}$ est inversible.

Comme dans le cas différentiel, un système $(\star)$ peut avoir un système fondamental de solutions de la forme précédente sans être de première espèce. On dit alors que $(\star)$ est fuchsien en $+\infty$.

Notons $g l\left(\mu, \mathcal{K}_{f}\right)$ l'anneau des matrices qui s'écrivent $A(x)=A_{1}(x)+$ $A_{2}(x)$ où $A_{2}(x)$ vérifie une condition $(C, \lambda)$ et $A_{1}(x)$ est un polynôme sans terme constant, à coefficients dans $g l(\mu, \mathbf{C})$. On note $\mathrm{GL}\left(\mu, \mathcal{K}_{f}\right)$ le groupe des éléments inversibles de $g l\left(\mu, \mathcal{K}_{f}\right)$.

Définition 1.4. Soit $A(x) \in g l\left(\mu, \mathcal{K}_{f}\right)$ et $T(x) \in \operatorname{GL}\left(\mu, \mathcal{K}_{f}\right)$. On définit

$$
A^{T}(x)=T(x-1)^{-1}\left(A(x) T(x)-(x-1){ }_{-1}^{\Delta} T(x)\right) .
$$

Les systèmes de matrice $A(x)$ et $B(x)$ sont dits $\mathcal{K}_{f}$-équivalents s'il existe $T(x) \in \mathrm{GL}\left(\mu, \mathcal{K}_{f}\right)$ telle que $B(x)=A^{T}(x)$.

Cette définition traduit le fait que $X(x)=T(x) Y(x)$ est une solution du système $(\star)$ de matrice $A(x)$ si et seulement si $Y(x)$ est une solution du système $(\star)$ de matrice $A^{T}(x)$.

On vérifie que, si $T_{1}, T_{2} \in \mathrm{GL}\left(\mu, \mathcal{K}_{f}\right), A^{T_{1} T_{2}}(x)=\left(A^{T_{1}}\right)^{T_{2}}(x)$ et que, si $T \in \mathrm{GL}(\mu, \mathbf{C})$, alors $A^{T}(x)=T^{-1} A(x) T$.

Toujours de façon parallèle au cas différentiel, le résultat classique qui suit ([6]) montre qu'il suffit d'étudier le cas d'un système de première espèce.

Proposition 1.5. Le système $(\star)$ est fuchsien en $+\infty$ si et seulement s'il est $\mathcal{K}_{f}$-équivalent à un système de première espèce.

Le système fondamental de solutions indiqué dans la proposition 1.3 fait intervenir à côté des séries de factorielles les mêmes "caractères", $x^{\lambda}$ et $\ln x$, que ceux que l'on utilise dans le cas différentiel. Pour un opérateur aux 
$q$-différences J. Sauloy a remarqué qu'il était possible d'utiliser des fonctions méromorphes dans $\mathbf{C}^{*}$, donc sans monodromie. Nous reprenons cette idée pour les équations aux différences et indiquons au paragraphe 2 comment résoudre un système $(\star)$ de matrice constante en utilisant des fonctions méromorphes dans $\mathbf{C}$, définies à partir de la fonction $\Gamma$ et qui sont limites lorsque $q \rightarrow 1$ de celles utilisées dans [7].

1.2. Choix de la $\boldsymbol{q}$-déformation. Le cas régulier correspond à un sytème de première espèce pour lequel $A_{0}=0$. Ce cas est étudié en [1] en supposant que $q$ est un réel $>1$. Le choix de $q$ réel est probablement technique mais c'est seulement sous cette hypothèse que nous avons obtenu les résultats que nous réutiliserons ici. C'est pourquoi dans toute la suite on suppose $q>1$ et on pose $p=1 / q$.

Guidé par le cas régulier, lui-même inspiré de la confluence de

$$
\delta_{q} f(x)=\frac{f(q x)-f(x)}{(q-1) x}
$$

vers $d f / d x$ quand $q \rightarrow 1$, on déforme $(\star)$ en un système de l'un des deux types suivants.

- Dans le plan des $x$, on considère un système

$$
p(x-1) \frac{X(p x-p)-X(x)}{(p-1) x-p}=A_{q}(x) X(x)
$$

où $A_{q}(x)$ est une série de factorielles mixtes, c'est-à-dire une série

$$
A_{q}(x)=\sum_{s \geq 0} A_{s}(q) e_{s}^{(q)}(x)
$$

avec $A_{s}(q) \in g l(\mu, \mathbf{C}), e_{0}^{(q)}(x)=1$ et, si $s \geq 1$,

$$
e_{s}^{(q)}(x)=\frac{1}{x(q x+1)\left(q^{2} x+[2]_{q}\right) \cdots\left(q^{s-1} x+[s-1]_{q}\right)} .
$$

Dans cette formule, on a utilisé la notation de Jackson

$$
[z]_{q}=\frac{q^{z}-1}{q-1}
$$

Une condition suffisante de convergence est (voir [1]) l'existence de $M>0$ et $\lambda>0$ tels que pour tout $s \geq 0,\left\|A_{s}(q)\right\| \leq M / e_{s}^{(q)}(\lambda)$. La série converge alors dans le domaine

$$
\left|x-\frac{1}{1-q}\right|>\lambda-\frac{1}{1-q} .
$$

Remarquons que l'homographie $\sigma_{p,-p}(x)=p x-p$ qui intervient dans $(\star)_{q}$ est l'inverse de $\sigma_{q, 1}$. 
- Dans le plan des $t$ défini par le changement de variable (homographique) $x=(t-1) /(q-1)$, ce qui revient à normaliser l'homographie $\sigma_{q, 1}$ en $\sigma_{q}$, le système $(\star)_{q}$ se transcrit en

$$
(p t-1) \delta_{p} Y(t)=\widetilde{A}_{q}(t) Y(t)
$$

si on a posé $Y(t)=X\left(\frac{t-1}{q-1}\right)$.

Cette fois $\widetilde{A}_{q}(t)$ est une série de $q$-factorielles, c'est-à-dire une série

$$
\widetilde{A}_{q}(t)=\sum_{s \geq 0} \frac{\widetilde{A}_{s}(q)}{(t ; q)_{s}}
$$

avec $(t ; q)_{0}=1$ et, pour $s \geq 1$,

$$
(t ; q)_{s}=(1-t)(1-q t) \cdots\left(1-q^{s-1} t\right) .
$$

Les séries de $q$-factorielles sont étudiées dans [1] où il est constaté qu'elles sont une autre façon d'écrire les séries convergentes à l'infini. La confluence s'obtient en faisant le changement de variable $t=q^{u}$ de sorte que $x=[u]_{q}$ et que l'application définissant le changement de variable du plan des $x$ vers celui des $u$ tend vers l'identité.

\section{Système constant}

On étudie dans ce paragraphe le cas du système

$$
(x-1) \underset{-1}{\Delta} X(x)=A X(x)
$$

où $A \in g l(\mu, \mathbf{C})$, que l'on déforme, selon la première possibilité, en

$$
p(x-1) \frac{X(p x-p)-X(x)}{(p-1) x-p}=A(q) X(x)
$$

où $A(q) \in g l(\mu, \mathbf{C})$ sera précisée ci-dessous et vérifiera $\lim _{q \rightarrow 1} A(q)=A$.

On peut aussi écrire l'équation $(\star)_{q}$ sous la forme

$$
X(x)=\left(B(q)-\frac{1}{x} A(q)\right) X(q x+1)
$$

avec $B(q)=I_{\mu}-(q-1) A(q)$ qui est une matrice inversible si $q$ est assez proche de 1.

On traitera successivement le cas où la matrice $A$ est semi-simple, le cas où elle possède un seul bloc de Jordan et enfin le cas général. 
2.1. Cas semi-simple. Remarquons d'abord que, lorsque $\mu=1$, l'équation $(\star)$ est l'équation des caractères :

$$
(x-1){ }_{-1}^{\Delta} f(x)=\alpha f(x)
$$

où $\alpha \in \mathbf{C}$. Le cas où $\alpha$ est entier est étudié dans $[\mathbf{1}]$. On la déforme en $(\star)_{q}$ avec $A(q)=-[-\alpha]_{q}$. On est ainsi conduit à utiliser la déformation suivante d'une matrice semi-simple.

Définition 2.1. Soit $A \in g l(\mu, \mathbf{C})$ une matrice semi-simple. On note $S \in$ $\mathrm{GL}(\mu, \mathbf{C})$ et $\alpha_{1}, \cdots, \alpha_{\mu}$ des nombres complexes non nécessairement distincts tels que $A=S^{-1} \operatorname{diag}\left(\alpha_{1}, \cdots, \alpha_{\mu}\right) S$.

Pour $q \neq 1$, soit $S(q) \in \mathrm{GL}(\mu, \mathbf{C})$ telle que $\lim _{q \rightarrow 1} S(q)=S$. On appelle système $q$-déformé du système $(\star)$ le système $(\star)_{q}$ de matrice

$$
A(q)=S(q)^{-1} \operatorname{diag}\left(-\left[-\alpha_{1}\right]_{q}, \cdots,-\left[-\alpha_{\mu}\right]_{q}\right) S(q) .
$$

La matrice $B(q)$ intervenant dans la forme $(*)_{q}$ est alors

$$
B(q)=S(q)^{-1} \operatorname{diag}\left(q^{-\alpha_{1}}, \cdots, q^{-\alpha_{\mu}}\right) S(q)
$$

qui est inversible pour tout $q$ assez proche de 1.

Avant d'étudier le système $(\star)_{q}$, nous commençons par quelques rappels classiques ou tirés de [7].

Soit $\alpha \in \mathbf{C}$. Une fonction $z(x)$ est solution de l'équation

$$
\left(1-\frac{[\alpha]_{q}}{x}\right) z(q x+1)=q^{\alpha} z(x)
$$

si et seulement si la fonction $g(t)=z\left(\frac{t-1}{q-1}\right)$ vérifie

$$
\left(t-q^{\alpha}\right) g(q t)=q^{\alpha}(t-1) g(t) .
$$

Puisque $\lim _{t \rightarrow 0} q^{\alpha}(t-1) /\left(t-q^{\alpha}\right)=1$, le point 0 est un point régulier pour l'équation $(\diamond)$ qui admet la solution méromorphe sur $\mathbf{C}$ :

$$
g_{\alpha}(t)=\frac{(p t ; p)_{\infty}}{\left(p^{\alpha+1} t ; p\right)_{\infty}}
$$

où $(\xi ; p)_{\infty}=\prod_{k=0}^{\infty}\left(1-p^{k} \xi\right)$ pour $\xi \in \mathbf{C}$.

Rappelons que la fonction entière $(\xi ; p)_{\infty}$ vérifie la relation fonctionnelle

$$
(q \xi ; p)_{\infty}=(1-q \xi)(\xi ; p)_{\infty}
$$

et s'annule aux points $\xi=q^{n}, n \in \mathbf{N}$.

Si $\alpha \notin \mathbf{Z}$, la fonction $g_{\alpha}(t)$ a des pôles simples aux points $t=q^{n+\alpha}$, $n \in \mathbf{N}^{*}$ et des zéros simples aux points $t=q^{n}, n \in \mathbf{N}^{*}$.

Si $\alpha$ est un entier positif, $g_{\alpha}$ est le polynôme $(1-p t)\left(1-p^{2} t\right) \cdots\left(1-p^{\alpha} t\right)$ et si $\alpha$ est un entier négatif ou nul, $g_{\alpha}(t)=1 /(t ; q)_{-\alpha}$. 
On peut aussi étudier $(\diamond)$ en considérant le point $\infty$ qui est singulier régulier puisque $\lim _{t \rightarrow \infty} q^{\alpha}(t-1) /\left(t-q^{\alpha}\right)=q^{\alpha} \in \mathbf{C}^{*}$.

Suivant [7], la solution méromorphe dans $\mathbf{C}^{*}$, obtenue "en partant de l'infini", est $g_{\alpha}(t) p(t)$ où ([7] p. 1060)

$$
p(t)=e_{q^{-\alpha}}\left(\frac{1}{t}\right) \frac{q^{\alpha} \Theta_{q}\left(q^{-\alpha} t\right)}{\Theta_{q}(t)}
$$

où $\Theta_{q}$ est la fonction de Jacobi définie par

$$
\Theta_{q}(t)=\sum_{n \in \mathbf{Z}}(-1)^{n} q^{-\frac{n(n-1)}{2}} t^{n}
$$

et où, pour $c \in \mathbf{C}^{*}, e_{c}(t)=\frac{\Theta_{q}(t)}{\Theta_{q}\left(c^{-1} t\right)}$.

La fonction de Jacobi vérifie $\Theta_{q}\left(\frac{1}{t}\right)=-\frac{1}{t} \Theta_{q}(t)$ et donc

$$
p(t)=\frac{q^{\alpha} \Theta_{q}\left(\frac{1}{t}\right) \Theta_{q}\left(q^{-\alpha} t\right)}{\Theta_{q}(t) \Theta_{q}\left(\frac{q^{\alpha}}{t}\right)}=\frac{q^{\alpha}\left(-\frac{1}{t}\right)}{\left(-\frac{q^{\alpha}}{t}\right)}=1 .
$$

Autrement dit $g_{\alpha}(t)$ est aussi la solution obtenue à partir du point $\infty$.

En posant $t=q^{u}$, et donc $x=[u]_{q}$, on a

$$
g_{\alpha}\left(q^{u}\right)=(1-p)^{\alpha} \frac{\Gamma_{p}\left(1+\alpha_{j}-u\right)}{\Gamma_{p}(1-u)}
$$

où $\Gamma_{p}$ est la fonction gamma $p$-analogue de Jackson ([5] par exemple).

Lemme 2.2. Soit $\alpha \in \mathbf{C}$, $q$ un réel $>1$ et $p=\frac{1}{q}$. On pose

$$
f_{\alpha}(x)=\frac{\Gamma(1+\alpha-x)}{\Gamma(1-x)}
$$

et

$$
z_{\alpha}^{(q)}(x)=(1-p)^{-\alpha} \frac{((1-p) x+p ; p)_{\infty}}{\left(p^{\alpha}((1-p) x+p) ; p\right)_{\infty}} .
$$

Ces fonctions sont méromorphes sur $\mathbf{C}$ et vérifient

$$
\begin{aligned}
f_{\alpha}(x) & =\left(1-\frac{\alpha}{x}\right) f_{\alpha}(x+1), \\
z_{\alpha}^{(q)}(x) & =\left(q^{-\alpha}+\frac{[-\alpha]_{q}}{x}\right) z_{\alpha}^{(q)}(q x+1) .
\end{aligned}
$$

Uniformément sur tout compact de $\mathbf{C} \backslash\left\{\right.$ pôles de $\left.f_{\alpha}\right\}$, on a

$$
\lim _{q \rightarrow 1^{+}} z_{\alpha}^{(q)}(x)=f_{\alpha}(x) \text {. }
$$


Preuve. On sait ([5]) que lorsque $p \rightarrow 1^{-}$, la fonction $\Gamma_{p}$ tend vers la fonction $\Gamma$, uniformément sur tout compact de $\mathbf{C} \backslash-\mathbf{N}$. Or

$$
z_{\alpha}^{(q)}(x)=(1-p)^{-\alpha} g_{\alpha}((q-1) x+1)
$$

et le facteur de normalisation est choisi pour que la limite existe. Les autres affirmations sont claires.

Remarquons que, si $\alpha \notin \mathbf{Z}, f_{\alpha}$ a des pôles simples aux points $x \in \alpha+\mathbf{N}^{*}$ et des zéros aux points $x \in \mathbf{N}^{*}$. Si $\alpha$ est un entier $>0, f_{\alpha}$ est le polynôme $(1-x)(2-x) \cdots(\alpha-x)$ et si $\alpha$ est un entier négatif ou nul, $f_{\alpha}(x)=x^{-[-\alpha]}$.

Avec les notations de la définition 2.1 et en remarquant que, si $X(x)$ est solution de $(\star)_{q}$ et donc de $(*)_{q}$, la $j$-ième composante du vecteur $Z(x)=$ $S(q) X(x)$ vérifie l'équation

$$
\left(1-\frac{1}{x}\left[\alpha_{j}\right]_{q}\right) z(q x+1)=q^{\alpha_{j}} z(x)
$$

à laquelle on applique le lemme 2.2, on obtient le résultat suivant.

Proposition 2.3. Soit $A \in g l(\mu, \mathbf{C})$ une matrice semi-simple et $(\star)_{q}$ un sytème q-déformé du système $(\star)$ de matrice A. Avec les notations du lemme 2.2, la matrice

$$
\mathcal{E}^{(q)}(x)=S(q)^{-1} \operatorname{diag}\left(z_{\alpha_{1}}^{(q)}(x), \cdots, z_{\alpha_{\mu}}^{(q)}(x)\right)
$$

est méromorphe dans $\mathbf{C}$, à pôles simples appartenant à $\bigcup_{j=1}^{\mu}\left[\alpha_{j}+\mathbf{N}^{*}\right]_{q}$. Elle constitue une matrice fondamentale de solutions de $(\star)_{q}$.

Lorsque $q \rightarrow 1^{+}, \mathcal{E}^{(q)}(x)$ converge, uniformément sur tout compact de $\mathbf{C} \backslash \bigcup_{j=1}^{\mu}\left(\alpha_{j}+\mathbf{N}^{*}\right)$, vers la matrice

$$
\mathcal{E}(x)=S^{-1} \operatorname{diag}\left(f_{\alpha_{1}}(x), \cdots, f_{\alpha_{\mu}}(x)\right)
$$

qui est une matrice fondamentale de solutions du système $(\star)$, constituée de fonctions méromorphes dans $\mathbf{C}$, à pôles simples appartenant à $\bigcup_{j=1}^{\mu}\left(\alpha_{j}+\mathbf{N}^{*}\right)$. Si $\alpha_{j} \in \mathbf{Z}$, la demi-ligne de pôles correspondante est absente si $\alpha_{j} \geq 0$ et remplacée par un ensemble fini de $-\alpha_{j}$ points si $\alpha_{j}<0$.

2.2. Cas d'un seul bloc de Jordan. On suppose maintenant que $\mu \geq 2$ et $A=\alpha I_{\mu}+N_{\mu}$ où $I_{\mu}$ est la matrice identité et $N_{\mu}$ la matrice nilpotente :

$$
N_{\mu}=\left(\begin{array}{ccccc}
0 & 1 & 0 & \cdots & 0 \\
0 & 0 & 1 & \cdots & 0 \\
\vdots & & & & \vdots \\
\vdots & & & 0 & 1 \\
0 & 0 & \cdots & 0 & 0
\end{array}\right)
$$

de sorte que pour tout entier $k, N_{\mu}^{k}=0$ si et seulement si $k \geq \mu$.

Pour obtenir un système fondamental de solutions du système

$$
(x-1){ }_{-1} X(x)=\left(\alpha I_{\mu}+N_{\mu}\right) X(x)
$$


on utilise des "logarithmes" associés à la famille $f_{\alpha}(x)$ de caractères définie au paragraphe précédent. Il s'agit ([2]) de la suite de fonctions définie pour $k \in \mathbf{N}$ par

$$
\psi_{k}(x)=\frac{1}{k !} \frac{\Gamma^{(k)}}{\Gamma}(1-x)
$$

où $\Gamma^{(k)}$ désigne la dérivée $k$-ième de la fonction $\Gamma$. Cette suite de fonctions vérifie $\psi_{0}(x)=1$ et, pour tout $k \in \mathbf{N}^{*}$,

$$
(x-1){ }_{-1}^{\Delta} \psi_{k}(x)=\psi_{k-1}(x) .
$$

Une translation de la variable conduit au lemme suivant.

Lemme 2.4. Pour $\alpha \in \mathbf{C}$ et $k \in \mathbf{N}$, posons $\psi_{k, \alpha}(x)=\psi_{k}(x-\alpha)$. Alors, pour tout $k \geq 1$,

$$
\psi_{k, \alpha}(x+1)-\psi_{k, \alpha}(x)=\frac{1}{x-\alpha} \psi_{k-1, \alpha}(x+1)
$$

et la matrice

$$
\mathcal{L}_{\mu, \alpha}(x)=f_{\alpha}(x)\left(\begin{array}{ccccc}
1 & \psi_{1, \alpha}(x) & \psi_{2, \alpha}(x) & \cdots & \psi_{\mu-1, \alpha}(x) \\
0 & 1 & \psi_{1, \alpha}(x) & \cdots & \psi_{\mu-2, \alpha}(x) \\
\vdots & & & & \vdots \\
\vdots & & & & \psi_{1, \alpha}(x) \\
0 & 0 & \ldots & 0 & 1
\end{array}\right)
$$

est un système fondamental de solutions du système

$$
(x-1){ }_{-1}^{\Delta} X(x)=\left(\alpha I_{\mu}+N_{\mu}\right) X(x) .
$$

On déforme $A$ en $A(q)=-[-\alpha]_{q} I_{\mu}+\varphi_{\alpha}(q) N_{\mu}$ où $\lim _{q \rightarrow 1^{+}} \varphi_{\alpha}(q)=1$. Le vecteur $V(x)$ défini par l'égalité $X(x)=z_{\alpha}^{(q)}(x) V(x)$ vérifie l'équation

$$
V(x)=\left(I_{\mu}-\frac{(q-1) \varphi_{\alpha}(q)((q-1) x+1)}{q^{-\alpha}((q-1) x+1)-1} N_{\mu}\right) V(q x+1) .
$$

En choisissant $\varphi_{\alpha}(q)=q^{-\alpha} \ln q /(q-1)$ et en posant $x=[u]_{q}$ et $G(u)=$ $V\left([u]_{q}\right)$, on obtient l'équation

$$
G(u)=\left(I_{\mu}-\frac{\ln q}{1-q^{\alpha-u}} N_{\mu}\right) G(u+1) .
$$

Or, on a le résultat suivant $([\mathbf{2}])$.

Lemme 2.5. La suite de fonctions définie pour $k \in \mathbf{N}$ par

$$
L_{k}(u)=\frac{(-1)^{k} \ln ^{k} q}{k ! \Gamma_{p}(1-u)}\left(\frac{d^{k}}{d c^{k}} \Gamma_{p}\left(1-u-\frac{\ln c}{\ln q}\right)\right)_{\left.\right|_{c=1}}
$$


vérifie $L_{0}(u)=1$ et pour $k \in \mathbf{N}^{*}$,

$$
L_{k}(u+1)-L_{k}(u)=\frac{\ln q}{1-q^{-u}} L_{k-1}(u+1) .
$$

Preuve. La famille de fonctions définie pour $c$ voisin de 1 par

$$
g_{c}(u)=\frac{\Gamma_{p}\left(1-u-\frac{\ln c}{\ln q}\right)}{\Gamma_{p}(1-u)}
$$

vérifie $g_{1}(u)=1$ et

$$
\left(c q^{u}-1\right) g_{c}(u+1)=\left(q^{u}-1\right) g_{c}(u)
$$

qui traduit la relation fonctionnelle de la fonction $\Gamma_{p}$. En dérivant $k$ fois cette relation par rapport à $c$ puis en donnant à $c$ la valeur 1 , on obtient le résultat annoncé.

On déduit de ce lemme le résultat suivant.

Proposition 2.6. Posons

$$
u_{q}(x)=\frac{\ln (1+(q-1) x)}{\ln q} \quad \text { et } \quad \ell_{k, \alpha}^{(q)}(x)=L_{k}\left(u_{q}(x)-\alpha\right) .
$$

La matrice

$$
\mathcal{L}_{\mu, \alpha}^{(q)}(x)=z_{\alpha}^{(q)}(x)\left(\begin{array}{ccccc}
1 & \ell_{1, \alpha}^{(q)}(x) & \ell_{2, \alpha}^{(q)}(x) & \cdots & \ell_{\mu-1, \alpha}^{(q)}(x) \\
0 & 1 & \ell_{1, \alpha}^{(q)}(x) & \cdots & \ell_{\mu-2, \alpha}^{(q)}(x) \\
\vdots & & & & \vdots \\
\vdots & & & & 1 \\
0 & 0 & \cdots & 0 & \ell_{1, \alpha}^{(q)}(x) \\
0 & & & & 1
\end{array}\right)
$$

constitue un système fondamental de solutions du système

$$
p(x-1) \frac{X(p x-p)-X(x)}{(p-1) x-p}=\left(-[-\alpha]_{q} I_{\mu}+q^{-\alpha} \frac{\ln q}{q-1} N_{\mu}\right) X(x)
$$

Ajoutons que pour tout compact $K$ de $\mathbf{C}$, il existe un réel $q_{K}>1$ tel que si $x \in K$ et $1<q<q_{K}$ alors $|(q-1) x|<1$. La fonction $u_{q}(x)$ est alors bien définie et holomorphe au voisinage de $K$ et $\mathcal{L}_{\mu, \alpha}^{(q)}(x)$ est méromorphe dans $\mathbf{C}$, à pôles d'ordre $\leq \mu$ appartenant à $\left[\alpha+\mathbf{N}^{*}\right]_{q}$.

Pour étudier le comportement à la limite, on peut comparer cette famille de "logarithmes" à une autre famille, pour laquelle le passage à la limite est classique $([2])$.

Lemme 2.7. La suite de fonctions définie pour $k \in \mathbf{N}$ par

$$
\widetilde{L}_{k}(u)=\frac{1}{k !}\left(\frac{d^{k}}{d c^{k}} \frac{\Gamma_{p}(1-u+c)}{\Gamma_{p}(1-u)}\right)_{\left.\right|_{c=0}}
$$


vérifie $\widetilde{L}_{0}(u)=1$ et pour $k \geq 1$,

$$
\widetilde{L}_{k}(u+1)-\widetilde{L}_{k}(u)=\frac{1}{1-q^{-u}} \sum_{j=1}^{k} \frac{(-1)^{j-1}}{j !} \ln ^{j} q \widetilde{L}_{k-j}(u+1) .
$$

De plus

$$
\lim _{q \rightarrow 1^{+}} \widetilde{L}_{k}(u)=\psi_{k}(u)
$$

uniformément sur tout compact de $\mathbf{C} \backslash \mathbf{N}^{*}$.

Le lemme suivant donne le lien entre les fonctions $L_{k}$ et $\widetilde{L}_{k}$.

Lemme 2.8. On a $L_{1}(u)=\widetilde{L}_{1}(u)$ et, pour $k \geq 2$,

$$
L_{k}(u)=\widetilde{L}_{k}(u)+\sum_{j=1}^{k-1} a_{k, j} \ln ^{j} q \widetilde{L}_{k-j}(u)
$$

où les $a_{k, j}$ sont des nombres rationnels indépendants de $q$.

Preuve. On dérive $k$ fois par rapport à $\lambda$ la relation

$$
\Gamma_{p}\left(1-u-\frac{\ln \lambda}{\ln q}\right)=\Gamma_{p}(1-u+c) \circ c(\lambda)
$$

où la fonction $c(\lambda)=-\ln \lambda / \ln q$ vérifie $c(1)=0$ et, pour $k \geq 1$,

$$
c^{(k)}(1)=\frac{(-1)^{k}(k-1) !}{\ln q}
$$

La relation entre les dérivées est du type

$$
\frac{d^{k}}{d \lambda^{k}} \Gamma_{p}\left(1-u-\frac{\ln \lambda}{\ln q}\right)=\sum_{j=1}^{k} \frac{d^{j}}{d c^{j}} \Gamma_{p}(1-u+c) P_{j}\left(c^{\prime}, c^{\prime \prime}, \cdots, c^{(k-j+1)}\right)
$$

où $P_{j}$ est une somme à coefficients entiers positifs de termes de la forme $\left(c^{\prime}\right)^{n_{1}}\left(c^{\prime \prime}\right)^{n_{2}} \cdots\left(c^{(k-j+1)}\right)^{n_{k-j+1}}$ vérifiant

$$
n_{1}+n_{2}+\cdots n_{k-j+1}=j \quad \text { et } \quad n_{1}+2 n_{2}+\cdots+(k-j+1) n_{k-j+1}=k .
$$

Le résultat s'obtient en donnant à $\lambda$ la valeur 1 .

Corollaire 2.9. Lorsque $q \rightarrow 1^{+}$, la fonction $\ell_{k, \alpha}^{(q)}(x)$ converge vers $\psi_{k, \alpha}$, uniformément sur tout compact de $\mathbf{C} \backslash\left(\alpha+\mathbf{N}^{*}\right)$.

La proposition suivante dresse le bilan du cas où il y a un seul bloc de Jordan.

Proposition 2.10. Soit $\alpha \in C$ et $A=\alpha I_{\mu}+N_{\mu}$ avec $\mu \geq 2$. Posons

$$
A(q)=-[-\alpha]_{q} I_{\mu}+q^{-\alpha} \frac{\ln q}{q-1} N_{\mu}
$$


La matrice $\mathcal{L}_{\mu, \alpha}^{(q)}(x)$, définie dans la proposition 2.6, est méromorphe dans $\mathbf{C}$, à pôles d'ordre $\leq \mu$ et appartenant à $\left[\alpha+\mathbf{N}^{*}\right]_{q}$. Elle constitue une matrice fondamentale de solutions du système

$$
p(x-1) \frac{X(p x-p)-X(x)}{(p-1) x-p}=A(q) X(x) .
$$

Lorsque $q \rightarrow 1^{+}$, la matrice $\mathcal{L}_{\mu, \alpha}^{(q)}(x)$ converge, uniformément sur tout compact de $\mathbf{C} \backslash\left(\alpha+\mathbf{N}^{*}\right)$, vers la matrice $\mathcal{L}_{\mu, \alpha}(x)$, définie dans le lemme 2.4 . C'est une matrice fondamentale de solutions du système

$$
(x-1){ }_{-1} X(x)=\left(\alpha I_{\mu}+N_{\mu}\right) X(x)
$$

constituée de fonctions méromorphes dans $\mathbf{C}$, à pôles d'ordre $\leq \mu$ et appartenant $\grave{a} \alpha+\mathbf{N}^{*}$.

2.3. Cas général. La mise en commun de ces briques de base conduit au théorème suivant dans lequel on utilise les notations du lemme 2.4 et des propositions 2.3 et 2.6 .

Théorème 2.11. Soit $A \in g l(\mu, \mathbf{C})$. Il existe $S \in \mathrm{GL}(\mu, \mathbf{C})$, des complexes $\alpha_{1}, \cdots, \alpha_{r}$ et des entiers naturels non nuls $\mu_{1}, \cdots, \mu_{r}$ tels que $\sum_{j=1}^{r} \mu_{j}=\mu$ et $A=S^{-1} \operatorname{diag}\left(A^{(1)}, \cdots, A^{(r)}\right) S$ où, pour $j=1, \cdots, r$,

$$
A^{(j)}= \begin{cases}\alpha_{j} & \text { si } \mu_{j}=1 \\ \alpha_{j} I_{\mu_{j}}+N_{\mu_{j}} & \text { si } \mu_{j} \geq 2\end{cases}
$$

Soit $(S(q))_{q>1}$ une famille de matrices appartenant à $\mathrm{GL}(\mu, \mathbf{C})$ vérifiant $\lim _{q \rightarrow 1^{+}} S(q)=S$. Posons

$$
A(q)=S(q)^{-1} \operatorname{diag}\left(A^{(1)}(q), \cdots, A^{(r)}(q)\right) S(q)
$$

ò̀, pour $j=1, \cdots r$,

$$
A^{(j)}(q)= \begin{cases}-\left[-\alpha_{j}\right]_{q} & \text { si } \mu_{j}=1 \\ -\left[-\alpha_{j}\right]_{q} I_{\mu_{j}}+q^{-\alpha_{j}} \frac{\ln q}{q-1} N_{\mu_{j}} & \text { si } \mu_{j} \geq 2 .\end{cases}
$$

Alors la matrice

$$
\mathcal{E}_{A}^{(q)}(x)=S(q)^{-1} \operatorname{diag}\left(\mathcal{E}_{q}^{(1)}(x), \cdots, \mathcal{E}_{q}^{(r)}(x)\right),
$$

où $\mathcal{E}_{q}^{(j)}(x)=z_{\alpha_{j}}^{(q)}(x)$ si $\mu_{j}=1$ et $\mathcal{E}_{q}^{(j)}(x)=\mathcal{L}_{\mu_{j}, \alpha_{j}}^{(q)}(x)$ si $\mu_{j} \geq 2$, constitue une matrice fondamentale de solutions du système

$$
p(x-1) \frac{X(p x-p)-X(x)}{(p-1) x-p}=A(q) X(x) .
$$

La matrice $\mathcal{E}_{A}^{(q)}(x)$ est formée de fonctions méromorphes dans $\mathbf{C}$ dont les pôles appartiennent à $\bigcup_{j=1}^{r}\left(\left[\alpha_{j}\right]_{q}+\mathbf{N}^{*}\right)$. Un pôle de la forme $k+\left[\alpha_{j}\right]_{q}$ est d'ordre $\leq \mu_{j}$. 
Lorsque $q \rightarrow 1^{+}$, la matrice $\mathcal{E}_{A}^{(q)}(x)$ converge, uniformément sur tout compact de $\mathbf{C} \backslash \bigcup_{j=1}^{\mu}\left(\alpha_{j}+\mathbf{N}^{*}\right)$, vers la matrice

$$
\mathcal{E}_{A}(x)=S^{-1} \operatorname{diag}\left(\mathcal{E}^{(1)}(x), \cdots, \mathcal{E}^{(r)}(x)\right)
$$

où $\mathcal{E}^{(j)}=f_{\alpha_{j}}(x)$ si $\mu_{j}=1$ et $\mathcal{E}^{(j)}=\mathcal{L}_{\mu_{j}, \alpha_{j}}(x)$ si $\mu_{j} \geq 2$. La convergence est uniforme sur tout compact de $\mathbf{C} \backslash \bigcup_{j=1}^{r}\left(\alpha_{j}+\mathbf{N}^{*}\right)$. Si $\alpha_{j} \in \mathbf{Z}$ et $\mu_{j}=1$, la ligne de pôles correspondante est absente si $\alpha_{j} \geq 0$ et remplacée par un ensemble fini de $-\alpha_{j}$ points si $\alpha_{j}<0$. La matrice $\mathcal{E}_{A}(x)$ est une matrice fondamentale de solutions du système

$$
(x-1){ }_{-1}^{\Delta} X(x)=A X(x)
$$

constituée de fonctions méromorphes dans $\mathbf{C}$ dont les pôles appartiennent à $\bigcup_{j=1}^{r}\left(\alpha_{j}+\mathbf{N}^{*}\right)$. Un pôle de la forme $k+\alpha_{j}$ est d'ordre $\leq \mu_{j}$.

\section{Système de première espèce}

On établira au paragraphe 3.1 qu'un système $(\star)$ de première espèce de matrice $A(x)$ est $\mathcal{K}_{f}$ - équivalent au système de matrice constante $A_{0}=$ $A(\infty)$ lorsque celle-ci est non résonnante. Nous rappelons la définition de ce mot et le résultat, établi par exemple dans [3], qui permet, par une transformation polynomiale en $1 / x$, de regrouper toutes les valeurs propres différant d'un entier en une seule valeur propre multiple.

Définition 3.1. Un système $(x-1) \Delta X(x)=A(x) X(x)$, dont la matrice $A(x)=\sum_{s \geq 0} A_{s} x^{-[s]}$ est une série de factorielles convergente, est dit non résonnant si les différences de deux valeurs propres distinctes de la matrice $A_{0}$ ne sont pas entières.

Proposition 3.2. Appelons $\alpha_{1}, \cdots, \alpha_{r}$ les valeurs propres distinctes de la matrice $A_{0}$. Il existe une transformation $T(x)=T_{0}+(1 / x) T_{1}$, où $T_{0}$ et $T_{1}$ sont des matrices constantes, telle que, si $\widetilde{A}(x)=A^{T}(x)$, les valeurs propres de $\widetilde{A}_{0}$ sont $\alpha_{1}+1, \alpha_{2}, \cdots, \alpha_{r}$.

Nous supposons donc dans la suite que le système $(\star)$ est de première espèce et non résonnant. Remarquons qu'alors, si $A_{0}(q)$ est la déformation de $A_{0}$ définie dans le théorème 2.11 et si $q$ est assez proche de 1 , le quotient de deux valeurs propres distinctes de la matrice

$$
B_{0}(q)=I_{\mu}-(q-1) A_{0}(q)
$$

n'appartient pas à $q^{\mathbf{Z}}$.

Ce dernier paragraphe est composé de deux parties. Dans la première nous établissons que tout système non résonnant est équivalent au système constant de matrice $A(\infty)$. La deuxième partie traite le même problème pour le système $q$-déformé et donne un théorème de confluence. 
3.1. Solution canonique dans le cas non résonnant. On établit tout d'abord la proposition suivante qui généralise celle établie dans [1] dans le cas régulier.

Proposition 3.3. Soit $(\star)$ un système non résonnant vérifiant la condition $(C, \lambda)$. On suppose que 0 est valeur propre de $A_{0}$. Pour tout vecteur $U_{0}$ appartenant au noyau de $A_{0}$, le système $(\star)$ admet une unique solution formelle $X(x)=U_{0}+\sum_{s \geq 1} X_{s} x^{-[s]}$. De plus, si $b=\sup _{s \geq 1}\left\|\left(s I_{\mu}+A_{0}\right)^{-1}\right\|$, $X(x)$ vérifie la condition $\left(b C\left\|U_{0}\right\|, \lambda+b C\right)$. En particulier $X(x)$ converge pour $\Re x>\lambda+b C$.

Preuve. Si $X(x)=U_{0}+\sum_{s \geq 1} X_{s} x^{-[s]}$ avec $X_{s} \in \mathbf{C}^{m}$, alors

$$
(x-1) \underset{-1}{\Delta} X(x)=-\sum_{s \geq 1} s X_{s} x^{-[s]}
$$

D'autre part

$$
A(x) X(x)=A_{0} U_{0}+\sum_{s \geq 1}\left(A_{0} X_{s}+A_{s} U_{0}+\sum_{(j, \ell, k) \in J_{s}} c_{j, \ell}^{(k)} A_{j} X_{\ell}\right) x^{-[s]} .
$$

Puisque $A_{0} U_{0}=0$, le problème formel équivaut à la liste de relations, pour $s \geq 1$ :

$$
\left(s I_{\mu}+A_{0}\right) X_{s}+A_{s} U_{0}+\sum_{(j, \ell, k) \in J_{s}} c_{j, \ell}^{(k)} A_{j} X_{\ell}=0
$$

où $J_{1}=\emptyset$ et pour $s \geq 2$ :

$$
J_{s}=\{j+\ell+k=s, j \geq 1, \ell \geq 1, k \geq 0\}
$$

et

$$
c_{j, \ell}^{(k)}=\frac{(j+k-1) !(\ell+k-1) !}{k !(j-1) !(\ell-1) !} .
$$

Ces relations définissent de manière unique les $X_{s}$ par récurrence puisque, pour tout $s \geq 1$, la matrice $s I_{\mu}+A_{0}$ est inversible.

On pose $u_{0}=\left\|U_{0}\right\|$ et pour $s \geq 1, a_{s}=\left\|A_{s}\right\|$ et $\xi_{s}=\left\|X_{s}\right\|$. Par hypothèse la série $\sum_{s>1} a_{s} x^{-[s]}$ converge pour $\Re x>\lambda$. Notons $a(x)$ sa somme.

La suite $\left\|\left(s I_{\mu}+A_{0}\right)^{-1}\right\|$ tend vers 0 quand $s \rightarrow \infty$ et donc $b$ est fini. Le fait que les $c_{j, \ell}^{(k)}$ sont positifs permet d'obtenir la suite d'inégalités valables pour $s \geq 1$,

$$
\xi_{s} \leq b\left(a_{s} u_{0}+\sum_{(j, \ell, k) \in J_{s}} c_{j, \ell}^{(k)} a_{j} \xi_{\ell}\right) .
$$

Par récurrence on définit $\bar{\xi}_{1}=b a_{1} u_{0}$ et, pour $s \geq 2$,

$$
\bar{\xi}_{s}=b\left(a_{s} u_{0}+\sum_{(j, \ell, k) \in J_{s}} c_{j, \ell}^{(k)} a_{j} \bar{\xi}_{\ell}\right)
$$


de sorte que $0 \leq \xi_{s} \leq \bar{\xi}_{s}$ pour $s \geq 1$. Notons $\chi(x)$ la somme (au moins formelle) de la série $\sum_{s \geq 1} \bar{\xi}_{s} x^{-[s]}$. Elle vérifie l'équation

$$
\chi(x)=b \sum_{s \geq 1}\left(a_{s} u_{0}+\sum_{(j, \ell, k) \in J_{s}} c_{j, \ell}^{(k)} a_{j} \bar{\xi}_{\ell}\right) x^{-[s]}=b\left(u_{0} a(x)+a(x) \chi(x)\right)
$$

ou encore

$$
\chi(x)=\frac{b u_{0} a(x)}{1-b a(x)}=-u_{0}\left(1-\frac{1}{1-b a(x)}\right) .
$$

La proposition 2.1 de [1] fournit alors le résultat.

Théorème 3.4. Soit $(\star)$ un système non résonnant. Il existe une unique transformation formelle tangente à l'identité

$$
F(x)=I_{\mu}+\sum_{s \geq 1} F_{s} x^{-[s]}
$$

telle que $A^{F}(x)=A_{0}$. De plus $F(x)$ vérifie une condition $\left(C^{\prime}, \lambda^{\prime}\right)$ et converge donc dans un demi-plan $\Re x \gg 0$.

Preuve. Établissons d'abord l'existence d'une unique série formelle $F(x)=$ $I_{\mu}+\sum_{s \geq 1} F_{s} x^{-[s]}$ vérifiant l'égalité

$$
A(x) F(x)-(x-1) \Delta_{-1}^{\Delta} F(x)=F(x-1) A_{0} .
$$

D'une part,

$$
(x-1) \underset{-1}{\Delta} F(x)=-\sum_{s \geq 1} s F_{s} x^{-[s]}
$$

et d'autre part, en utilisant la classique formule de translation, on a

$$
F(x-1)=I_{\mu}+\sum_{s \geq 1}\left(F_{s}+(s-1) ! \sum_{k=1}^{s-1} \frac{F_{k}}{(k-1) !}\right) x^{-[s]} .
$$

Pour $M, N \in g l(\mu, \mathbf{C})$, notons $\Phi_{M, N}$ l'endomorphisme défini sur $g l(\mu, \mathbf{C})$ par $\Phi_{M, N}(U)=M U-U N$. L'équation (*) équivaut alors à la liste de relations

$$
\Phi_{A_{0}+I_{\mu}, A_{0}}\left(F_{1}\right)=-A_{1}
$$

et pour $s \geq 2$,

$$
\Phi_{A_{0}+s I_{\mu}, A_{0}}\left(F_{s}\right)=-A_{s}+(s-1) ! \sum_{k=1}^{s-1} \frac{F_{k} A_{0}}{(k-1) !}-\sum_{(j, \ell, k) \in J_{s}} c_{j, \ell}^{(k)} A_{j} F_{\ell} .
$$

Dans le cas non résonnant, pour $s \geq 1$, les matrices $A_{0}+s I_{\mu}$ et $A_{0}$ n'ont pas de valeur propre commune et $\Phi_{A_{0}+s I_{\mu}, A_{0}}$ est un isomorphisme. On en déduit par récurrence l'existence et l'unicité de la suite $\left(F_{s}\right)$. 
Pour établir la convergence de cette série de factorielles, on interprète $(*)$ comme un système de dimension $\mu^{2}$ auquel on peut appliquer la proposition 3.3. Pour cela on remarque que $(*)$ s'écrit aussi

$$
\begin{aligned}
(x-1) \Delta_{-1}^{\Delta} F(x)= & \left(A(x) F(x)-F(x) A_{0}\right)\left(I_{\mu}-\frac{A_{0}}{x-1}\right)^{-1} \\
= & \left(A_{0} F(x)-F(x) A_{0}+\sum_{s \geq 1} A_{s} F(x) x^{-[s]}\right) \\
& \quad \times\left(I_{\mu}+\sum_{s \geq 1} A_{0}\left(A_{0}+I_{\mu}\right) \cdots\left(A_{0}+(s-1) I_{\mu}\right) x^{-[s]}\right) \\
= & \sum_{s \geq 0} \mathcal{A}_{s}(F(x)) x^{-[s]}
\end{aligned}
$$

où $\mathcal{A}_{s}$ est la suite d'opérateurs linéaires définis sur l'espace vectoriel $g l(\mu, \mathbf{C})$ par $\mathcal{A}_{0}(U)=A_{0} U-U A_{0}, \mathcal{A}_{1}(U)=A_{1} U+\left(A_{0} U-U A_{0}\right) A_{0}$ et pour $s \geq 2$,

$$
\mathcal{A}_{s}(U)=A_{s} U+\left(A_{0} U-U A_{0}\right) B_{s}+\sum_{(j, \ell, k) \in J_{s}} c_{j, \ell}^{(k)} A_{j} U B_{\ell}
$$

où on a posé, pour $s \geq 1, B_{s}=A_{0}\left(A_{0}+I_{\mu}\right) \cdots\left(A_{0}+(s-1) I_{\mu}\right)$.

L'opérateur $\mathcal{A}_{0}$ admet 0 pour valeur propre et $U=I_{\mu}$ est un vecteur propre pour cette valeur propre. L'hypothèse de non résonnance implique qu'aucun entier non nul n'est valeur propre de $\mathcal{A}_{0}$.

D'autre part, si $A(x)$ vérifie la condition $(C, \lambda)$ et si $a_{0}=\left\|A_{0}\right\|$, la norme de l'opérateur $\mathcal{A}_{s}$ se majore par

$$
K_{s}=C \frac{\Gamma(\lambda+s-1)}{\Gamma(\lambda)}+2 a_{0} \frac{\Gamma\left(a_{0}+s\right)}{\Gamma\left(a_{0}\right)}+C \sum_{(j, \ell, k) \in J_{s}} c_{j, \ell}^{(k)} \frac{\Gamma\left(a_{0}+j\right)}{\Gamma\left(a_{0}\right)} \frac{\Gamma(\lambda+\ell-1)}{\Gamma(\lambda)} .
$$

En exprimant que les séries de factorielles obtenues en développant les deux fonctions

$$
1-\frac{a_{0}-\lambda+1}{x-\lambda} \text { et } 1+\frac{a_{0}-\lambda+1}{x-a_{0}-1}
$$

sont inverses l'une de l'autre, on obtient la formule ([1] p. 343)

$$
\left(a_{0}-\lambda+1\right) \sum_{(j, \ell, k) \in J_{s}} c_{j, \ell}^{(k)} \frac{\Gamma\left(a_{0}+j\right)}{\Gamma\left(a_{0}+1\right)} \frac{\Gamma(\lambda+\ell-1)}{\Gamma(\lambda)}=\frac{\Gamma\left(a_{0}+s\right)}{\Gamma\left(a_{0}+1\right)}-\frac{\Gamma(\lambda+s-1)}{\Gamma(\lambda)} .
$$

On en déduit :

$$
K_{s}=C \frac{1-\lambda}{a_{0}+1-\lambda} \frac{\Gamma(\lambda+s-1)}{\Gamma(\lambda)}+\left(2 a_{0}+\frac{C}{a_{0}+1-\lambda}\right) \frac{\Gamma\left(a_{0}+s\right)}{\Gamma\left(a_{0}\right)} .
$$

En posant $\widetilde{\lambda}=\max \left(\lambda, a_{0}+1\right)$, on prouve l'existence d'une constante $\widetilde{C}>0$ telle que $K_{s} \leq \widetilde{C} \Gamma(\widetilde{\lambda}+s-1) / \Gamma(\widetilde{\lambda})$, ce qui permet d'appliquer la proposition 3.3. 
Le système $(\star)$ admet donc un système fondamental de solutions de la forme $F(x) \mathcal{E}_{A_{0}}(x)$ où $F(x)$ est l'unique solution de $A^{F}(x)=A_{0}$ telle que $F(\infty)=I_{\mu}$ et $\mathcal{E}_{A_{0}}(x)$, défini dans le théorème 2.11 , dépend de la forme normale de Jordan $J_{0}$ de $A_{0}$ mais aussi du choix d'une matrice $S$ conjuguant $A_{0}$ à $J_{0}$. La forme normale $J_{0}$ est unique lorsqu'on a fixé l'ordre de ses blocs et la matrice $\mathcal{E}_{J_{0}}(x)$ est alors clairement définie. On va voir que $(\star)$ admet un système fondamental de solutions indépendant du choix de la matrice $S$. Cette propriété repose sur les deux lemmes suivants.

Lemme 3.5. Soit $S \in \mathrm{GL}(\mu, \mathbf{C})$. La matrice $F(x) S^{-1} \mathcal{E}_{J_{0}}(x)$ est un système fondamental de solutions de $(\star)$ si et seulement si $J_{0} S=S A_{0}$.

Preuve. La définition de $\mathcal{E}_{A_{0}}(x)$ montre que $\mathcal{E}_{A_{0}}(x)=S^{-1} \mathcal{E}_{J_{0}}(x)$ si $S \in$ $\mathrm{GL}(\mu, \mathbf{C})$ a été choisie telle que $J_{0} S=S A_{0}$. Inversement si $S \in \mathrm{GL}(\mu, \mathbf{C})$ est telle que $F(x) S^{-1} \mathcal{E}_{J_{0}}(x)$ soit une solution de $(\star)$, alors on doit avoir $A^{F S^{-1}}=$ $J_{0}$. Puisque $A^{F}=A_{0}$, on en déduit $A_{0}^{S^{-1}}=J_{0}$ ou encore $S A_{0} S^{-1}=J_{0}$.

Lemme 3.6. Toute matrice constante qui commute avec $J_{0}$ commute avec $\mathcal{E}_{J_{0}}(x)$.

Preuve. Reprenons les notations du théorème 2.11 et supposons que

$$
J_{0}=\operatorname{diag}\left(A^{(1)}, \cdots, A^{(r)}\right)
$$

où chaque $A^{(j)}$ est un bloc de Jordan élémentaire de dimension $\mu_{j}$, de la forme $A^{(j)}=\alpha_{j}$ si $\mu_{j}=1$ et $A^{(j)}=\alpha_{j} I_{\mu_{j}}+N_{\mu_{j}}$ si $\mu_{j} \geq 2$. Partitionnons toute matrice constante $S$ selon cette décomposition en blocs élémentaires : $S=\left(S_{j, h}\right)_{1 \leq j, h \leq r}$. On sait que $S$ commute avec $J_{0}$ si et seulement si chaque bloc vérifie :

- $S_{j, h}=0$ si $\alpha_{j} \neq \alpha_{h}$

- $N_{\mu_{j}} S_{j, h}=S_{j, h} N_{\mu_{h}}$ si $\alpha_{j}=\alpha_{h}$.

La matrice $\mathcal{E}_{J_{0}}(x)$ a la même structure en blocs diagonaux que $J_{0}$ et le bloc d'indice $j$ est

$$
\mathcal{E}^{(j)}(x)=f_{\alpha_{j}}(x)\left(I_{\mu_{j}}+\sum_{k=1}^{\mu_{j}-1} \psi_{k, \alpha_{j}}(x) N_{\mu_{j}}^{k}\right) .
$$

La condition de commutation de $S$ et de $\mathcal{E}_{J_{0}}(x)$ s'écrit

$$
S_{j, h} \mathcal{E}^{(h)}(x)=\mathcal{E}^{(j)}(x) S_{j, h}
$$

pour $j, h=1, \cdots, r$. Ces relations sont vérifiées pour les indices tels que $\alpha_{j} \neq \alpha_{h}$ puisqu'alors $S_{j, h}=0$. Lorsque $\alpha_{j}=\alpha_{h}=\alpha$, la condition s'écrit

$$
S_{j h}+\sum_{k=1}^{\mu_{h}-1} \psi_{k, \alpha}(x) S_{j h} N_{\mu_{h}}^{k}=S_{j h}+\sum_{k=1}^{\mu_{j}-1} \psi_{k, \alpha}(x) N_{\mu_{j}}^{k} S_{j h}
$$


ou encore en remarquant que $N_{\mu_{h}}^{k}=0$ pour $k \geq \mu_{h}$ et $N_{\mu_{j}}^{k}=0$ pour $k \geq \mu_{j}$,

$$
\sum_{k \geq 1} \psi_{k, \alpha}(x) S_{j h} N_{\mu_{h}}^{k}=\sum_{k \geq 1} \psi_{k, \alpha}(x) N_{\mu_{j}}^{k} S_{j h}
$$

égalité qui est assurée par les relations $N_{\mu_{j}} S_{j h}=S_{j h} N_{\mu_{h}}$.

Théorème 3.7. Soit $(\star)$ un système de première espèce et $A_{0}=A(\infty)$. Soit $F(x)$ la solution de $A^{F}=A_{0}$ donnée par le théorème 3.4. Soit $J_{0}$ une forme de Jordan de $A_{0}$ et $\mathcal{E}_{J_{0}}(x)$ la matrice qui lui est associée dans le théorème 2.11. La matrice $F(x) S^{-1} \mathcal{E}_{J_{0}}(x) S$ est une matrice fondamentale de solutions de $(\star)$, indépendante du choix de $S \in \mathrm{GL}(\mu, \mathbf{C})$ vérfiant $J_{0} S=$ $S A_{0}$.

Preuve. Le lemme 3.5 et le fait qu'en multipliant à droite une matrice de solutions par une matrice constante on obtient une matrice de solutions montrent que si $S_{1}$ et $S_{2}$ vérifient la condition indiquée, $F(x) S_{i}^{-1} \mathcal{E}_{J_{0}}(x) S_{i}$ $(i=1,2)$ est une matrice fondamentale de solutions de $(\star)$. Puisque la matrice $S=S_{1} S_{2}^{-1}$ commute avec $J_{0}$, le lemme 3.6 permet d'écrire

$$
S_{1}^{-1} \mathcal{E}_{J_{0}}(x) S_{1}=S_{1}^{-1} \mathcal{E}_{J_{0}}(x) S_{1} S_{2}^{-1} S_{2}=S_{1}^{-1} S_{1} S_{2}^{-1} \mathcal{E}_{J_{0}}(x) S_{2}=S_{2}^{-1} \mathcal{E}_{J_{0}}(x) S_{2}
$$

et le résultat s'en déduit par multiplication à gauche par $F(x)$.

Définition 3.8. On appelle solution canonique du système $(\star)$ et on note $\mathcal{X}_{\text {can }}(x)$ la matrice fondamentale de solutions décrite dans le théorème 3.7 .

3.2. Système $q$-déformé et confluence. On indique maintenant comment choisir un système $q$-déformé d'un système $(\star)$ non résonnant. Cette étude est faite dans le plan de la variable $t=(q-1) x+1$ où le système aux $q$-différences obtenu est du type étudié dans [7]. Nous aurons cependant besoin de reprendre en partie les résultats classiques de façon à pouvoir traiter la confluence à l'aide des théorèmes de [1].

On déforme le système $(\star)$ en un système

$$
(p t-1) \delta_{p} Y(t)=A_{q}(t) Y(t)
$$

où $A_{q}(t)=\sum_{s \geq 0} \frac{A_{s}(q)}{(t ; q)_{s}}$ est une série de $q$-factorielles convergente.

L'équation $(\widetilde{\star})_{q}$ peut aussi s'écrire

$$
Y(t)=B_{q}(t) Y(q t)
$$

où

$$
B_{q}(t)=I_{\mu}+\frac{(1-q) t}{t-1} A_{q}(q t) .
$$

Puisque la somme d'une série de $q$-factorielles convergente est holomorphe à l'infini, si la matrice $B_{q}(\infty)=I_{\mu}+(1-q) A_{0}(q)$ est inversible, le système $(\widetilde{*})_{q}$ est fuchsien à l'infini au sens de [7]. Il est non résonnant, toujours 
au sens de [7], si le quotient de deux valeurs propres distinctes de $B_{0}(q)=$ $I_{\mu}-(q-1) A_{0}(q)$ n'appartient pas à $q^{\mathbf{Z}}$. Dans ce cas, on peut montrer ([7]) qu'il existe une unique matrice $F_{q}(t) \in \mathrm{GL}\left(\mu, \mathbf{C} \llbracket \frac{1}{t} \rrbracket\right)$ telle que $F_{q}(\infty)=I_{\mu}$ et $A_{q}^{F_{q}}(t)=A_{0}(q)$ où $A_{q}^{F_{q}}(t)=F_{q}(p t)^{-1}\left(A_{q}(t) F_{q}(t)-(p t-1) \delta_{p} F_{q}(t)\right)$ est la matrice du système obtenu à partir de $(\widetilde{\star})_{q}$ par le changement de fonction inconnue $Y(t)=F_{q}(t) Z(t)$. De plus la série $F_{q}(t)$ converge. Ce résultat permet de décrire un système fondamental de solutions de $(\widetilde{\star})_{q}$ pour $q$ fixé, mais pour obtenir des propriétés de confluence, il faut préciser la dépendance en $q$ des coefficients $A_{s}(q)$.

Supposons que la matrice $A(x)=\sum_{s \geq 0} A_{s} x^{-[s]}$ du système $(\star)$ à déformer vérifie la condition $(C, \lambda)$. Pour $A_{0}(q)$, on reprend les notations et hypothèses faites dans le théorème 2.11 en remplaçant $A$ par $A_{0}$. Si le sytème $(\star)$ est non résonnant tout système $(*)_{q}$ dont la matrice $A_{q}(t)$ a pour terme constant $A_{0}(q)$ est alors non résonnant si $q$ est assez proche de 1 . On suppose ensuite que les coefficients $A_{s}(q), s \geq 1$, vérifient les hypothèses suivantes (voir $[\mathbf{1}])$ :

1) il existe $q_{0}>1$ tel que si $1<q<q_{0}$, alors

$$
\left\|A_{s}(q)\right\| \leq\left(q^{C}-1\right) q^{s+\lambda-1}\left|\left(q^{\lambda} ; q\right)_{s-1}\right|,
$$

2) $\lim _{q \rightarrow 1^{+}}(1-q)^{-s} A_{s}(q)=A_{s}$.

On suppose $q_{0}$ assez petit pour que $B_{0}(q)$ soit non résonnante pour $1<$ $q<q_{0}$ et on résume toutes ces hypothèses en disant que le système $(\widetilde{\star})_{q}$ est obtenu par $q$-déformation du système $(\star)$. On suit une démarche analogue à celle du paragraphe 3.1 et on commence par établir le $q$-analogue suivant de la proposition 3.3, sous des hypothèses restrictives mais suffisantes pour notre étude.

Proposition 3.9. Soit $(\widetilde{\star})_{q}$ un système obtenu par q-déformation d'un système $(\star)$ non résonnant dont la matrice $A_{0}$ admet 0 pour valeur propre. On suppose qu'il existe un vecteur $U_{0}$, indépendant de $q$, appartenant au noyau de $A_{0}(q)$ pour tout $q$ assez proche de 1 . Le système $(\widetilde{\star})_{q}$ admet alors, pour $q$ assez proche de 1 , une unique solution formelle

$$
Y(t)=U_{0}+\sum_{s \geq 1} \frac{Y_{s}(q)}{(t ; q)_{s}} .
$$

De plus il existe $C^{\prime}>0$ et $q_{0}>1$ tels que pour tout $s \geq 1$ et tout $q$ tel que $1<q \leq q_{0}$, on ait

$$
\left\|Y_{s}(q)\right\| \leq\left\|U_{0}\right\|\left(q^{C^{\prime}}-1\right) q^{s+\lambda+C^{\prime}-1}\left|\left(q^{\lambda+C^{\prime}} ; q\right)_{s-1}\right| .
$$

Preuve. On recopie celle de la proposition 3.3, en utilisant la série majorante

$$
\chi(t)=-u_{0}\left(1-\frac{1}{1-b(q) a_{q}(t)}\right)
$$


où $u_{0}=\left\|U_{0}\right\|, a_{q}(t)=\sum_{s>1}\left\|A_{s}(q)\right\| /(t ; q)_{s}$ et $b(q)$ majore la norme de toutes les matrices $\left([s]_{q} I_{\mu}+A_{0}(q)\right)^{-1}$. Pour estimer $b(q)$, on remarque que les valeurs propres de $\left([s]_{q} I_{\mu}+A_{0}(q)\right)^{-1}$ sont de la forme $(q-1) /\left(q^{s}-q^{-\alpha}\right)$ où $\alpha$ est une valeur propre de $A_{0}$, et peuvent se majorer, pour tout $q>1$, par 1 si $\Re \alpha>0$, par $(q-1) /\left(q^{b}-1\right)$ où $b=d\left(\Re \alpha,-\mathbf{N}^{*}\right)$ si $\Re \alpha \notin-\mathbf{N}^{*}$ et par la même expression avec $b<|\varepsilon|$ et pour $q \leq q_{0}$ si $\alpha=-s_{0}+i \varepsilon$ où $s_{0} \in \mathbf{N}^{*}$. On peut ensuite majorer la norme du bloc de taille $\nu$ correspondant à $\alpha$ : $(q-1)\left(\left(q^{s}-q^{-\alpha}\right) I_{\nu}+q^{-\alpha} \ln q N_{\nu}\right)^{-1}$ par

$$
\frac{q-1}{q^{b}-1} \frac{1-q^{-\nu \Re \alpha}}{1-q^{-\Re \alpha}}
$$

si $\Re \alpha \neq 0$ et par $\nu(q-1) /\left(q^{b}-1\right)$ si $\Re \alpha=0$. Ces estimations et l'hypothèse faite sur $a_{q}(t)$ permettent d'utiliser la proposition 4.5 de [1] pour conclure.

Donnons maintenant l'analogue de la proposition 3.4 en indiquant les modifications à apporter à sa preuve.

Proposition 3.10. Soit $(\widetilde{\star})_{q}$ un système obtenu par q-déformation d'un système $(\star)$ non résonnant. L'unique transformation tangente à l'identité $F_{q}(t)$ telle que $A_{q}^{F_{q}}(t)=A_{0}(q)$ admet un développement en série de qfactorielles $F_{q}(t)=I_{\mu}+\sum_{s \geq 1} \frac{F_{s}(q)}{(t ; q)_{s}}$ dont les coefficients vérifient les deux propriétés :

1) il existe $q_{0}>1, C^{\prime}, \lambda^{\prime}>0$ tels que pour tout $q$ avec $1<q<q_{0}$ et tout $s \geq 1$,

$$
\left\|F_{s}(q)\right\| \leq\left(q^{C^{\prime}}-1\right) q^{s+\lambda^{\prime}-1}\left|\left(q^{\lambda^{\prime}} ; q\right)_{s-1}\right|,
$$

2) $\lim _{q \rightarrow 1^{+}}(1-q)^{-s} F_{s}(q)=F_{s}$ où $F(x)=I_{\mu}+\sum_{s \geq 1} F_{s} x^{-[s]}$ est la série de factorielles du théorème 3.4 .

Preuve. Comme dans la preuve du théorème 3.4, la majoration s'obtient en appliquant la proposition 3.9 avec $U_{0}=I_{\mu}$ au système de dimension $\mu^{2}$ suivant qui exprime la condition $A_{q}^{F_{q}}(t)=A_{0}(q)$ :

$$
(p t-1) \delta_{p} F_{q}(t)=\left(A_{q}(t) F_{q}(t)-F_{q}(t) A_{0}(q)\right)\left(B_{0}(q)+\frac{1-q}{p t-1} A_{0}(q)\right)^{-1} .
$$

On a $\left(B_{0}(q)+\frac{1-q}{p t-1} A_{0}(q)\right)^{-1}=\sum_{s \geq 0} \frac{C_{s}(q)}{(t ; q)_{s}}$ où $C_{0}(q)=B_{0}(q)^{-1}$ et pour $s \geq 1$,

$$
C_{s}(q)=q^{s}(1-q)^{s} B_{0}(q)^{-s-1} A_{0}(q)\left(A_{0}(q)+I_{\mu}\right) \cdots\left(A_{0}(q)+[s-1]_{q} I_{\mu}\right) .
$$


On peut alors écrire

$$
(p t-1) \delta_{p} F_{q}(t)=\sum_{s \geq 0} \mathcal{A}_{s}(q)\left(F_{q}(t)\right) \frac{1}{(t ; q)_{s}}
$$

où $\mathcal{A}_{s}(q)$ est l'opérateur linéaire sur $g l(\mu, \mathbf{C})$ défini par

$$
\mathcal{A}_{0}(q)(U)=\left(A_{0}(q) U-U A_{0}(q)\right) B_{0}(q)^{-1}
$$

et, pour $s \geq 1$,

$$
\begin{aligned}
\mathcal{A}_{s}(q)(U)= & A_{s}(q) U B_{0}(q)^{-1}+\left(A_{0}(q) U-U A_{0}(q)\right) C_{s}(q) \\
& +\sum_{(j, \ell, k) \in J_{s}} c_{j, \ell}^{(k)}(q) A_{j}(q) U C_{\ell}(q) .
\end{aligned}
$$

où $J_{s}$ est l'ensemble d'indices défini dans la preuve de la proposition 3.3 et où

$$
c_{j, \ell}^{(k)}(q)=q^{k+j \ell}(1-q)^{k} \frac{[j+k-1] ![\ell+k-1] !}{[k] ![j-1] ![\ell-1] !}
$$

si on pose $[0] !=1$ et, pour $n \in N,[n] !=[1]_{q}[2]_{q} \cdots[n]_{q}$.

La deuxiéme hypothèse faite sur la suite $A_{s}(q)$ implique que pour $s \geq 1$, $\lim _{q \rightarrow 1}(1-q)^{-s} C_{s}(q)=B_{s}$ (notation du théorème 3.4). En utilisant le fait que

$$
\widetilde{c}_{j, \ell}^{(k)}(q)=c_{j, \ell}^{(k)}(q)(1-q)^{-k} \rightarrow c_{j, \ell}^{(k)}
$$

quand $q \rightarrow 1$, on vérifie que l'opérateur $(1-q)^{-s} \mathcal{A}_{s}(q)$ a pour limite l'opérateur $\mathcal{A}_{s}$.

Pour majorer la norme de $\mathcal{A}_{s}(q)$, on procède comme dans la preuve de 3.4 en utilisant le lemme 4.4 de [1]. Pour cela on remarque qu'en posant $D_{0}(q)=A_{0}(q) B_{0}(q)^{-1}$, chaque bloc de Jordan de $D_{0}(q)$ est de la forme

$$
[\alpha]_{q} I_{\nu}+\frac{q^{\alpha}}{q-1} \sum_{i=1}^{\nu-1} \ln ^{i} q N_{\nu}^{i}
$$

où $\alpha$ est une valeur propre de $A_{0}$ et $\nu$ la taille du bloc de Jordan correspondant. D'autre part, on peut écrire

$$
C_{s}(q)=q^{s}(1-q)^{s} B_{0}(q)^{-1} \prod_{k=0}^{s-1}\left(D_{0}(q)+[k]_{q} B_{0}(q)^{-1}\right)
$$

et remarquer que chaque bloc de Jordan de $D_{0}(q)+[k]_{q} B_{0}(q)^{-1}$ est de la forme

$$
[\alpha+k]_{q} I_{\nu}+\frac{q^{\alpha+k}}{q-1} \sum_{i=1}^{\nu-1} \ln ^{i} q N_{\nu}^{i} .
$$

En conclusion on énonce un théorème synthétisant l'étude faite dans le plan de la variable $x$ initiale, ce qui conduit (toujours selon [1]) à remplacer les séries de $q$-factorielles par les séries de factorielles mixtes et à modifier 
en conséquence les hypothèses demandées à une q-déformation. Le résultat final de convergence est une application du théorème 3.1 de [1].

Théorème 3.11. Soit

$$
(x-1){ }_{-1} X(x)=A(x) X(x)
$$

un système aux différences non résonnant dont la matrice

$$
A(x)=\sum_{s \geq 0} A_{s} x^{-[s]}
$$

vérifie la condition $(C, \lambda)$.

Soit $S \in \mathrm{GL}(\mu, \mathbf{C}), \alpha_{1}, \cdots, \alpha_{r} \in \mathbf{C}$ et $\mu_{1}, \cdots, \mu_{r} \in \mathbf{N}^{*}$ tels que $\sum_{j=1}^{r} \mu_{j}=$ $\mu$ et que, en posant, pour $j=1, \cdots, r$,

$$
A^{(j)}= \begin{cases}\alpha_{j} & \text { si } \mu_{j}=1 \\ \alpha_{j} I_{\mu_{j}}+N_{\mu_{j}} & \text { si } \mu_{j} \geq 2\end{cases}
$$

on ait

$$
A_{0}=S^{-1} \operatorname{diag}\left(A^{(1)}, \cdots, A^{(r)}\right) S .
$$

On note $\mathcal{X}_{\text {can }}(x)$ la solution canonique de $(\star)$.

Soit $(S(q))_{q>1}$ une famille de matrices appartenant à $\mathrm{GL}(\mu, \mathbf{C})$ vérifiant $\lim _{q \rightarrow 1^{+}} S(q)=S$. Posons, pour $q>1$,

$$
A_{0}(q)=S(q)^{-1} \operatorname{diag}\left(A^{(1)}(q), \cdots, A^{(r)}(q)\right) S(q)
$$

où, pour $j=1, \cdots r$,

$$
A^{(j)}(q)= \begin{cases}-\left[-\alpha_{j}\right]_{q} & \text { si } \mu_{j}=1, \\ -\left[-\alpha_{j}\right]_{q} I_{\mu_{j}}+q^{-\alpha_{j}} \frac{\ln q}{q-1} N_{\mu_{j}} & \text { si } \mu_{j} \geq 2 .\end{cases}
$$

Soit $A_{q}(x)=\sum_{s \geq 1} A_{s}(q) e_{s}^{(q)}(x)$ une série de factorielles mixtes telle que pour tout $s \geq 1$ :

Alors, il existe une unique série formelle de factorielles mixtes

$$
\mathcal{F}_{q}(x)=I_{\mu}+\sum_{s \geq 1} F_{s}(q) e_{s}^{(q)}(x)
$$

telle que, si $\mathcal{E}_{A_{0}}^{(q)}(x)$ est la matrice définie dans le théorème 2.11 , la matrice

$$
\mathcal{X}_{q}(x)=\mathcal{F}_{q}(x) \mathcal{E}_{A_{0}}^{(q)}(x) S(q)
$$

constitue un système fondamental de solutions du système

$$
p(x-1) \frac{X(p x-p)-X(x)}{(p-1) x-p}=\left(A_{0}(q)+A_{q}(x)\right) X(x) .
$$


De plus, il existe $\lambda^{\prime} \geq \lambda$ tel que la série $\mathcal{F}_{q}(x)$ converge pour

$$
\left|x-\frac{1}{1-q}\right|>\lambda^{\prime}-\frac{1}{1-q} .
$$

Lorsque $q \rightarrow 1^{+}, \mathcal{X}_{q}(x)$ converge vers $\mathcal{X}_{\text {can }}(x)$, uniformément sur tout compact de $\left\{\Re x \geq \lambda^{\prime}+1\right\} \backslash \bigcup_{j=1}^{r}\left(\alpha_{j}+\mathbf{N}^{*}\right)$.

Exemple. En dimension 1, l'équation

$$
(x-1) \underset{-1}{\Delta} y(x)=\left(a-\frac{\mu}{x-\lambda}\right) y(x)
$$

s'écrit

$$
y(x+1)=\frac{x(x+\lambda-1)}{\left(x-\mu_{1}\right)\left(x-\mu_{2}\right)} y(x)
$$

où $\mu_{1}+\mu_{2}=\lambda+a-1$ et $\mu_{1} \mu_{2}=\mu-a(1-\lambda)$. Le changement de fonction inconnue

$$
y(x)=\frac{\Gamma(1+a-x)}{\Gamma(1-x)} z(x)
$$

la transforme en

$$
z(x+1)=\frac{(x-a)(x+1-\lambda)}{\left(x-\mu_{1}\right)\left(x-\mu_{2}\right)} z(x)
$$

dont la solution, holomorphe dans un demi-plan $\Re x \gg 0$ et ayant 1 pour limite quand $x \rightarrow \infty$ dans ce demi-plan, est la fonction

$$
z_{+}(x)=\frac{\Gamma(x-a) \Gamma(x+1-\lambda)}{\Gamma\left(x-\mu_{1}\right) \Gamma\left(x-\mu_{2}\right)} .
$$

Cette fonction admet un développement en série de factorielles convergente qui peut s'obtenir en remarquant que la formule de Gauss-Kummer permet d'écrire pour $\Re x>\Re \lambda-1$,

$$
z_{+}(x)={ }_{2} F_{1}\left(\mu_{1}-a, \mu_{2}-a ; x-a ; 1\right) .
$$

Il suffit alors d'appliquer la formule de translation à cette série de factorielles en $x-a$.

La solution canonique de l'équation donnée est donc

$$
y_{+}(x)=\frac{\Gamma(1+a-x)}{\Gamma(1-x)} z_{+}(x) .
$$

Un résultat classique rappelé dans [4] permet de voir $z_{+}(x)$ comme limite quand $q \rightarrow 1^{+}$de ${ }_{2} \phi_{1}\left(q^{\mu_{1}-a}, q^{\mu_{2}-a}, q^{x-a} ; q ; q\right)$ où, par définition,

$$
{ }_{2} \phi_{1}(a, b, c ; q ; u)=\sum_{s \geq 0} \frac{(a ; q)_{s}(b ; q)_{s}}{c ; q)_{s}(q ; q)_{s}} u^{s}
$$


est une série convergente pour $|u|<|q c /(a b)|$. De plus, le $q$-analogue de la formule de Gauss-Kummer est la formule suivante (Jacobi et Heine, citée par [4]) :

$$
{ }_{2} \phi_{1}(a, b, c ; q ; q)=\frac{(a / c ; p)_{\infty}\left((b / c ; p)_{\infty}\right.}{(1 / c ; p)_{\infty}(a b / c ; p)_{\infty}} .
$$

Ces remarques, jointes à la proposition 2.3 , conduisent à considérer la fonction

$$
g_{q}(t)=\frac{(p t ; p)_{\infty}\left(q^{\mu_{1}} / t ; p\right)_{\infty}\left(q^{\mu_{2}} / t ; p\right)_{\infty}}{\left(q^{-a-1} t ; p\right)_{\infty}\left(q^{a} / t ; p\right)_{\infty}\left(q^{\lambda-1} / t ; p\right)_{\infty}}
$$

qui vérifie

$$
g_{q}(q t)=q^{a} \frac{(t-1)\left(t-q^{\lambda-1}\right)}{\left(t-q^{\mu_{1}}\right)\left(t-q^{\mu_{2}}\right)} g_{q}(t) .
$$

On en déduit

$$
(p t-1) \delta_{p} g_{q}(t)=A_{q}(t) g_{q}(t)
$$

où

$$
A_{q}(t)=q^{-a-1} \frac{\left(t-q^{\mu_{1}+1}\right)\left(t-q^{\mu_{2}+1}\right)}{(p-1) t\left(t-q^{\lambda}\right)}-\frac{p t-1}{(p-1) t}
$$

En tenant compte de le relation $\mu_{1}+\mu_{2}+1-a-\lambda=0$ dans la décomposition en éléments simples de $A_{q}(t)$, on trouve

$$
A_{q}(t)=-[-a]_{q}+\frac{\left(q^{\lambda-\mu_{1}-1}-1\right)\left(q^{\lambda-\mu_{2}-1}-1\right)}{(p-1)\left(t-q^{\lambda}\right)} .
$$

Dans le plan de la variable $x$, la fonction $f_{q}(x)=g_{q}((q-1) x+1)$ vérifie $p(x-1) \frac{f_{q}(p x-p)-f_{q}(x)}{(p-1) x-p}=\left(-[-a]_{q}+\frac{\left[\lambda-\mu_{1}-1\right]_{q}\left[\lambda-\mu_{2}-1\right]_{q}}{x-[\lambda]_{q}}\right) f_{q}(x)$.

On remarque que les conditions sur les paramètres impliquent la relation $\left(\lambda-\mu_{1}-1\right)\left(\lambda-\mu_{2}-1\right)=\mu$ et l'équation obtenue est clairement une déformation de l'équation initiale.

\section{References}

[1] A. Duval, Séries de q-factorielles, opérateurs aux q-différences et confluence, Annales de la Fac. des Sciences de Toulouse, 12 (2003), 335-374, MR 2030091.

[2] A. Duval, Une remarque sur les "logarithmes" associés à certains caractères, Aequationes Math., 68 (2004), 88-97.

[3] W.J. Fitzpatrick and L.J. Grimm, Convergent factorial series solutions of linear difference equations, J. Differential Equations, 29 (1978), 345-361, MR 0507483 (80f:39003), Zbl 0403.39001.

[4] G. Gasper, Elementary derivations of summation and transformation formulas for $q$-series, Special functions, $q$-series and related topics (Toronto, ON, 1995), Fields Inst. Commun., 14, Amer. Math. Soc., Providence, RI, 1997, 55-70, MR 1448679 (98f:33030), Zbl 0873.33013. 
[5] G. Gasper and M. Rahman, Basic Hypergeometric Series, Encyclopedia of Mathematics and its Applications, 35, Cambridge University Press, Cambridge, 1990, MR 1052153 (91d:33034), Zbl 0695.33001.

[6] W.A. Harris, Jr., Analytic theory of difference equations in 'Analytic theory of differential equations' (Proc. Conf., Western Michigan Univ., Kalamazoo, Mich., 1970), Lecture Notes in Mathematics, 183, Springer, Berlin 1971, 46-58, MR 0390565 (52 \#11390), Zbl 0232.39001.

[7] J. Sauloy, Systèmes aux q-différences singuliers réguliers : classification, matrice de connexion et monodromie, Ann. Inst. Fourier, 50 (2000), 1021-1071, MR 1799737 (2001m:39043), Zbl 0957.05012.

Received October 22, 2003 and revised April 13, 2004.

Laboratoire Paul Painleve

UMR-CNRS 8524

U.F.R. DE Mathématiques

59655 Villeneuve D'AsCQ

CEDEX

FRANCE

E-mail address: duval@math.univ-lille1.fr 